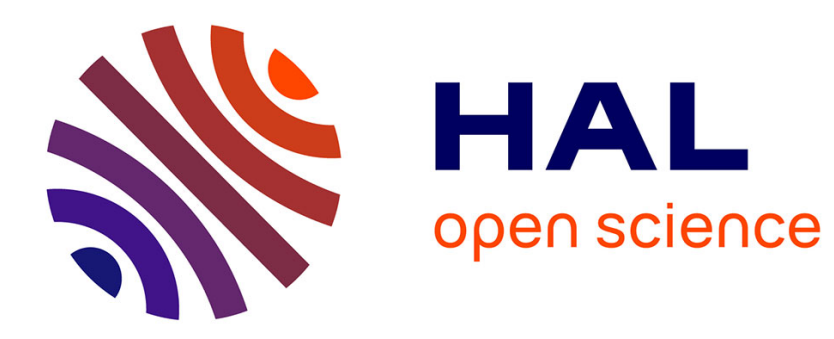

\title{
Les conflits du travail: enjeux scientifiques d'un phénomène global
}

Maxime Quijoux

\section{To cite this version:}

Maxime Quijoux. Les conflits du travail: enjeux scientifiques d'un phénomène global. Critique Internationale, 2014, Les conflits du travail dans le monde, 2014/3 (64), pp.9-16. hal-01545860

\section{HAL Id: hal-01545860 \\ https://hal.science/hal-01545860}

Submitted on 23 Jun 2017

HAL is a multi-disciplinary open access archive for the deposit and dissemination of scientific research documents, whether they are published or not. The documents may come from teaching and research institutions in France or abroad, or from public or private research centers.
L'archive ouverte pluridisciplinaire HAL, est destinée au dépôt et à la diffusion de documents scientifiques de niveau recherche, publiés ou non, émanant des établissements d'enseignement et de recherche français ou étrangers, des laboratoires publics ou privés. 
Les conflits du travail : enjeux scientifiques d'un phénomène global

par Maxime Quijoux

Il est des objets d'étude qui réclament plus que d'autres un effort d'attention et de construction alors même qu'ils sont en plein développement ou renouvellement. Les conflits du travail ${ }^{1}$ constituent un cas emblématique de ce paradoxe. Longtemps hégémonique dans le corpus des sciences sociales ${ }^{2}$, ce thème a connu à partir des années 1960 une certaine relégation, en France ${ }^{3}$ comme dans le reste du monde ${ }^{4}$. On a régulièrement expliqué cette désaffection par la diminution numérique et politique de la classe ouvrière au profit d'une société de services plus encline à la négociation ${ }^{5}$. Certes, il est difficile aujourd'hui «sur fond de crise et de recomposition du salariat, (...) de mobiliser les catégories de conflit et de négociation ${ }^{6}$ utilisées il y a soixante ans, mais le fait est que le regard de la recherche, lui aussi, a changé. Sociologues et politistes ont progressivement délaissé les mobilisations du travail au profit d'autres objets ou d'autres catégories de populations, y compris dans le cadre même des recherches sur le travail ou sur les mouvements sociaux ${ }^{7}$. Ainsi, depuis une dizaine d'années, certains travaux français montrent que, si la conflictualité n'a pas disparu des entreprises hexagonales, ses modalités d'expression se sont transformées : l'activité gréviste, par exemple, a perdu en durée et en intensité, tandis que d'autres formes d'expressions et de

1. Nous entendons par conflits du travail l'ensemble des formes de protestations qui s'expriment dans l'activité professionnelle ou l'emploi ou en rapport avec ceux-ci. S'il comprend les mobilisations syndicales, notre objet ne peut raisonnablement s'y limiter, étant donné l'absence ou la répression récurrente des organisations syndicales de par le monde. En revanche, la définition que Guy Groux donne du conflit du travail - polysémique, polyscénique et polymorphe - nous semble particulièrement bien adaptée à sa dimension internationale. Guy Groux, Vers un renouveau du conflit social ?, Paris, Bayard, 1998.

${ }^{2}$. Jean-Claude Javillier, Les conflits du travail, Paris, PUF, 1981 ; Roland Pfefferkorn Inégalités et rapports sociaux. Rapports de classes, rapports de sexes, Paris, La Dispute, 2007.

3. Richard Hyman, «À la recherche de la mobilisation perdue », dans Amélie Pouchet (dir.), Sociologie du travail : quarante ans après, Paris, Elsevier, 2001, p. 35-51.

${ }^{4}$. Voir, a minima, pour l'Amérique latine, Timothy Wickham-Crowley, Susan Eckstein, «Économie et sociologie politiques du militantisme et des répertoires des mouvements sociaux récents en Amérique latine », Revue internationale de politique comparée, 17 (2), 2010, p. 29-52 ; et pour l'Afrique, Jean Copans, «Pourquoi travail et travailleurs africains ne sont plus à la mode en 2014 dans les sciences sociales », Politique africaine, 133, 2014, p.25-44.

5 . Pour une synthèse de ces analyses, voir Baptiste Giraud, «Des conflits du travail à la sociologie des mobilisations : les apports d'un décloisonnement empirique et théorique », Politix, 86, 2009, p. 20. Voir aussi Leilla de Comarmond, Les vingt ans qui ont changé la CGT, Paris, Denoël Impacts, 2013 ou, dans un registre historique, Stéphane Sirot, Le syndicalisme, la politique et la grève (France et Europe, XIX ${ }^{e}-X X I^{e}$ siècles), Nancy, Éditions Arbre bleu, 2011.

6. Odile Join-Lambert et al., «Au-delà du conflit et de la négociation ? », dossier débat, Sociologie du travail, 53, 2011, p. 163.

7. Frédéric Sawicki, Johanna Siméant, «Décloisonner la sociologie de l'engagement militant. Note critique sur quelques tendances récentes des travaux français ». Sociologie du travail, 51 (1), 2009, p. 97-126; B. Giraud, «Des conflits du travail à la sociologie des mobilisations: les apports d'un décloisonnement empirique et théorique », art. cité. 
mobilisations professionnelles la remplaçaient ${ }^{8}$. Après avoir admis un temps l'idée d'un recul des mobilisations «matérialistes», en particulier autour de la question des salaires, en faveur d'engagements plus «qualitatifs », la science politique en France semble désormais plus prudente : au lieu de penser en termes de remplacement d'un type de mobilisation par un autre, elle admet la persistance, depuis les années 1990, du modèle ancien et reconnaît une porosité entre organisations associatives et salariales ${ }^{9}$.

Si elle est de plus en plus contestée dans le champ académique français, la thèse d'une pacification des relations de travail semble tout simplement inconcevable dès qu'on la porte au niveau international: depuis le milieu des années 1990, les litiges ne cessent d'augmenter dans les entreprises chinoises ${ }^{10}$, tandis que dans de nombreux pays et régions du monde les heurts et mobilisations ayant pour objet le travail sont de plus en plus forts ${ }^{11}$. L'actualité la plus récente

8. Jean-Michel Denis, Le conflit, en grève ?, Paris, La dispute, 2005 ; Sophie Béroud et al., La lutte
continue?, Paris, Les Éditions du Croquant, 2008.
9 . Érik Neveu, Sociologie des mouvements sociaux, Paris, La Découverte, 2011 ( $5^{\mathrm{e}}$ édition); Isabelle . Érik Neveu, Sociologie des mouvements sociaux, Paris, La Découverte, 2011 ( $5^{\mathrm{e}}$ édition) ; Isabelle
Sommier, «Vers une approche unifiée de la conflictualité sociale», et Jérôme Pélisse, «Ce que la juridicisation des relations de travail fait au conflit et à la négociation», dans O. Join-Lambert et al., «Audelà du conflit et de la négociation ? », cité, p. 176-180 et p. 185-190.

${ }^{10}$. En 1994, les conflits collectifs du travail étaient au nombre de 1482 et impliquaient 52637 travailleurs. En 2008, ils étaient au nombre de 21880 et impliquaient 502713 travailleurs, d'après l'Annuaire statistique du Travail chinois. Le nombre de travailleurs impliqués dans des conflits du travail individuels et collectifs est encore plus saisissant : sur la même période, il est passé de 77794 à 1214 328, c'est-à-dire qu'il a été multiplié par 15. Depuis, on a observé un certain reflux de la conflictualité puisque l'on ne comptabilisait plus « que » 7252 cas de conflits collectifs en 2011, soit près de 5 fois plus tout de même que vingt ans plus tôt. De fait, la situation « socioprofessionnelle » est encore très instable en Chine, comme en témoignent les rapports réguliers de l'ONG China Labor Watch ainsi que le dernier conflit en date, qui a engagé entre 30000 et 40000 ouvriers de chaussures de sport dans la province méridionale du Guangdong en avril 2014.

${ }^{11}$. Au Maroc, le nombre de grèves a doublé entre 2010 et 2011. D'après le ministère du Travail, il a atteint un record inégalé depuis dix ans avec plus de 350 mouvements. Depuis 2013, le Brésil connait des mouvements sociaux contre la vie chère auxquels ont succédé des mobilisations contre le coût de la Coupe du monde de football de 2014. À moins d'un mois de l'événement, les mobilisations se poursuivent et les appels à la grève se multiplient, aussi bien sur les chantiers des stades que dans les secteurs des transports, de l'éducation ou de la police. En Afrique du Sud, le 10 août 2012, les ouvriers de la mine de Marikana, dans le Nord du pays, se sont mis en grève pour réclamer des augmentations de salaire. Le 16 août, le conflit a dégénéré : les forces de l'ordre ont tiré sur les grévistes et provoqué la mort de 34 d'entre eux. Voir l'article de Raphaël Botiveau dans le volume 2 de ce dossier (65, octobre-décembre 2014). Le Cambodge a connu ses deux dernières années des grèves très suivies dans l'un de ses principaux secteurs d'activité, le textile, mais aussi des grèves générales lors des élections législatives de 2013. En Turquie, le 13 mai 2014, une explosion dans une mine de charbon à Soma, ville située dans l'Ouest du pays, a provoqué un incendie et l'effondrement des galeries. On a dénombré plus de 300 morts. Cette catastrophe a entraîné une série de manifestations, parfois violentes, contre le gouvernement accusé de laxisme sur les conditions et l'application des règles de sécurité. Le Bangladesh connaît depuis vingt ans des drames réguliers dans ses usines textiles, le plus grave étant celui du Rana Plaza, un immeuble situé près de Dacca qui s'est effondré le 24 avril 2013, entraînant la mort de plus de 1100 personnes et en blessant plus de 2000 . Depuis, le pays a connu toute une série de mobilisations, notamment au moment des élections qui ont été accompagnées d'un mouvement de grèves générales. Cet accident a également provoqué une mobilisation internationale aboutissant à une série d'actions spécifiques dont la signature d'un accord le 15 mai 2013 sur les conditions de travail et la mise au norme des usines dans le pays entre la fédération des industries européennes IndustriAll, Uni Global Union et d'une centaine de marques de vêtements. Sur le rôle du syndicalisme dans la révolution égyptienne, voir Françoise Clément $e t$ $a l$., «Le rôle des mobilisations des travailleurs et du mouvement syndical dans la chute de Moubarak », 
témoigne de cette agitation importante qui ne représente d'ailleurs que la partie la plus « visible »parce que la plus médiatique - de cette conflictualité sociale mondiale. Les pays «postindustriels », eux non plus, n'échappent pas aux mobilisations professionnelles, mais celles-ci peuvent prendre des formes différentes, voire nouvelles, dans leur genèse comme dans leur expression. Entre déclin des bastions industriels, libéralisation progressive des codes du travail et émergence du secteur tertiaire, les travailleurs et leurs organisations sont soumis à une séquence historique qui impose tout un travail d'adaptation souvent long et coûteux en termes de temps et d'énergie $^{12}$. De fait, parallèlement aux protestations régulières contre les restructurations, qui impliquent souvent des «communautés » de travail caractérisées par une forte tradition de luttes syndicales ${ }^{13}$, d'une part, aux grèves générales souvent massives provoquées par la crise financière de 2008 et par les politiques qu'elle a entrânées, d'autre part, les pratiques syndicales «traditionnelles » évoluent vers une professionnalisation par le droit ${ }^{14}$ et de nouveaux modes de mobilisations apparaissent progressivement dont certains sont investis par des populations jusque-là « invisibles ${ }^{15}$ et/ou inexpérimentées ${ }^{16}$.

Face à ces persistances et à ces évolutions, ce double dossier souhaite autant combler les lacunes actuelles de l'analyse qu'impulser une nouvelle dynamique de recherches. Dans les limites du format qui nous est imposé ici, et compte tenu du caractère encore très lacunaire des productions sur ce thème, il s'agit donc moins d'affirmer des thèses que de proposer des pistes de réflexion au regard des débats existants. À cet égard, les contributions réunies dans ces deux dossiers proposent trois problématiques particulièrement fécondes.

Causes et répertoires d'action : les vertus heuristiques et épistémologiques d'un décentrement

Mouvements, 66, 2011, p. 69-78. Sur la Tunisie, voir Allal Amin, « Réformes néolibérales, clientélismes et protestations en situation autoritaire », Politique africaine, 117, 2010, p. 107-125.

12. S. Béroud, J.-M. Denis, «Le développement interprofessionnel de Solidaires : entre volonté d'expérimentation et reproduction du "modèle" confédéral », La Revue de l'Ires, 75, 2012, p. 67-92.

13. Claude Didry, Annette Jobert (dir.), L'entreprise en restructuration. Dynamiques institutionnelles et mobilisations collectives, Rennes, Presses universitaires de Rennes, 2010; Nicolas Renahy, «Une occupation d'usine, chant du cygne d'un syndicalisme villageois », Ethnologie française, 35 (4), 2005, p. 691-702 ; Cédric Lomba, «Restructurations industrielles : appropriations et expropriations des savoirs ouvriers », Actes de la recherche en sciences sociales, 196-197, 2013, p. 34-53.

${ }^{14}$. Aristea Koukiadaki, Claude Didry, «Droit et conflits du travail dans l'Angleterre du New Labour », L'Homme et la société, 182, 2011, p. 11-23 ; J. Pélisse, «Judiciarisation ou juridicisation ?», Politix, 86, 2009, p. 73-96.

15. Mathieu Bonzom, «Syndicats et immigrés aux États-Unis », Plein droit, 89, 2011, p. 19-22; Pierre Barron et al., On bosse ici, on reste ici, la grève des sans-papiers, une aventure inédite, Paris, La Découverte, 2011.

16. Marlène Benquet, «Les raisons de l'action collective : retour sur la mobilisation improbable des salariés d'hypermarchés », Sociologie du travail, 52, 2010, p. 305-322 ; Catherine Sauviat, «Résurgence des luttes sociales et renouvellement des stratégies syndicales », Chronique internationale de l'IRES, 145, 2014, p. 317. 
La double dynamique d'affaiblissement de la classe ouvrière et de précarisation de nouvelles catégories de travailleurs qui est en cours depuis trente ans a conduit les chercheurs français en sciences sociales à reconsidérer les causes et les conditions de mobilisations dans le monde du travail. Après avoir renseigné les effets du déclin du monde ouvrier dans la chute des luttes au travail $^{17}$, la sociologie des mobilisations professionnelles s'intéresse depuis le début des années 2000 aux mobilisations qualifiées le plus souvent $d^{\prime}$ «improbables $»^{18}$. Parce qu'elles sont portées par des acteurs possédant peu de compétences politiques et une identité professionnelle évanescente, et qu'elles sont menées dans des conditions d'emploi et de travail instables, ces luttes bousculent les objets d'étude, les frontières disciplinaires et certaines routines analytiques sur les formes de mobilisations au travail. Compte tenu de la nature de certains régimes politiques et de l'absence dans certains pays de code du travail et/ou d'organisations syndicales, l'examen international des conflits du travail contribue, parfois de manière radicale, à déconstruire nos archétypes scientifiques souvent réduits à la mesure de la grève et au degré d'intégration salariale ${ }^{19}$. De fait, la pluralité et la richesse des répertoires d'action - du recours à l'émeute (Raphaël Botiveau) aux stratégies multiples de contournement des répressions syndicales (Quentin Delpech et Mathieu Hocquelet), en passant par l'appropriation de stratégies issues des mouvements sociaux (Sophie Béroud) ${ }^{20}$ ou par les campagnes transnationales $^{21}$ - permettent non seulement de mieux rendre compte des «évolutions des dynamiques des conflits, des négociations et des acteurs qui en sont à l'origine ${ }^{22}$, mais aussi de mettre en lumière leurs propriétés spécifiques. Porter un regard international sur les conflits du travail amène en outre à donner une autre épaisseur aux formes de circulation de ces répertoires ${ }^{23}$ et

17. Stéphane Beaud, Michel Pialoux, Retour sur la condition ouvrière, Paris, Fayard, 1999.

${ }^{18}$. Lilian Mathieu, «Les mobilisations improbables : pour une approche contextuelle et compréhensive », dans Stéphane Cadiou, Stéphanie Dechezelles, Antoine Roger, (dir.), Passer à l'action : les mobilisations émergentes, Paris, L'Harmattan, 2007 ; Annie Collovald, Lilian Mathieu, «Mobilisations improbables et apprentissage d'un répertoire syndical », Politix, 86, 2009, p. 119-143 ; Maxime Quijoux, «Mobilisations et politisations ouvrières contemporaines : les usines "récupérées" en Argentine », Critique internationale, 57, 2012, p. 111-132.

${ }^{19}$. B. Giraud, «Des conflits du travail à la sociologie des mobilisations : les apports d'un décloisonnement empirique et théorique », art. cité.

${ }^{20}$. Et C. Sauviat, « Résurgence des luttes sociales et renouvellement des stratégies syndicales », art. cité.

${ }^{21}$. Sur la construction des mobilisations transnationales relatives aux conditions de travail dans les usines textiles et sur la responsabilité sociale des entreprises, voir Pauline Barraud de Lagerie, «L'affaire Spectrum », Revue française de sociologie, 52 (2), 2011, p. 245-275. Pour un exemple très éclairant d'autres formes de mobilisations transnationales, voir le cas de la cyber-manifestation d'IBM rapportée par JeanEmmanuel Rey, « Nouveaux conflits collectifs dans notre société de réputation : de la grève interne à l'action collective externalisée », dans O. Join-Lambert et al., "Au-delà du conflit et de la négociation ? », cité, p. $170-175$.

${ }_{22}$. O. Join-Lambert et al., « Au-delà du conflit et de la négociation ?», cité, p. 162.

${ }^{23}$. Nous reprenons ici, en l'appliquant à un niveau mondial, l'idée d'« une démarche comparative sur les formes de circulation des personnes, des idées entre mondes patronal, syndical, associatif, étatique et politique ». Ibid., p. 164. 
aux liens qui unissent les différents acteurs de ces luttes, pour encore une fois dépasser nos schémas d'analyse habituels.

Repenser le champ des relations professionnelles

L'analyse des conflits du travail engage traditionnellement trois acteurs principaux : les travailleurs et/ou leurs représentants, les employeurs et les pouvoirs publics ${ }^{24}$. L'importance accordée à la production des règles et des institutions - de leur genèse à leurs effets sur le monde du travail - a amené la recherche à faire du droit et de la négociation des notions centrales-avec la grève et le conflit - des stratégies élaborées par ces différents acteurs ${ }^{25}$. Depuis le milieu des années 1980, les effets de la mondialisation des échanges et de l'émergence de nouveaux espaces mercantiles et institutionnels - l'Union européenne en premier lieu-, ont considérablement modifié cette approche. Dans la lignée des analyses du déclin des bastions industriels, de nombreux observateurs ont ainsi souligné l'incapacité des organisations syndicales à ajuster leurs schèmes d'analyses et leurs méthodes de lutte face à un capital de plus en plus volatile ${ }^{26}$. D'autres, en revanche, se sont efforcés de rendre compte des manières dont les différents acteurs tentaient de produire de nouvelles normes et actions au gré des différentes configurations économiques et politiques qui se constituaient progressivement ${ }^{27}$. De nouvelles règles et des dispositifs inédits ont vu le jour, renforçant par là même le passage d'un syndicalisme militant à un syndicalisme expert, voire technocratique $^{28}$. Une fois de plus, le décentrement autorisé par l'internationalisation de l'objet «conflit du travail » permet de dégager de nouvelles perspectives de recherche, et ce sur la base d'un constat préalable simple: de nombreux conflits du travail apparaissent dans des contextes politiques et sociaux où le système des relations professionnelles est inopérant, faute d'un marché formel du travail, faute d'État régulateur et/ou faute de partenaires sociaux indépendants ${ }^{29}$. Certes,

\footnotetext{
${ }^{24}$. Du moins pour la théorie du «système des relations professionnelles ». Antoine Bevort, Annette Jobert, Sociologie du travail, les relations professionnelles, Paris, Armand Colin, 2011.

${ }^{25}$. Ibid. ; Nicolas Hatzfeld, «Les conflits du travail, source renouvelée d'expérience et de connaissance », dans O. Join-Lambert et al., « Au-delà du conflit et de la négociation ? », cité, p.160-193.

${ }^{26}$. Georges Ubbiali, «Syndicalisme en crise, syndicalisme en mutation. Note critique », Politix, 6 (23), 1993, p. 84-101.

27. Donna Kesselman, «Travail et salariat aux États-Unis : quels droits, quelles perspectives ?», Revue française d'études américaines 111, 2007, p. 6-26 ; A. Koukiadaki, C. Didry, « Droit et conflits du travail dans l'Angleterre du New Labour», art. cité; Arnaud Mias, "Les registres de l'action syndicale européenne », Sociologie du travail, 51, 2009, p. 461-477.

28. Anne-Catherine Wagner, «La fabrique de syndicalistes "européens": une enquête sur les formations européennes de la CES », Politique européenne, 27, 2009, p. 105-127.

${ }^{29}$. Pour le Bangladesh, voir P. Barraud de Lagerie, «L'affaire Spectrum », art. cité ; pour la Chine, Claude Didry, Annette Jobert, Yi Zhenzhen dans ce dossier et Chloé Froissart dans la prochaine livraison (65, octobre-
} 
des tentatives d'institutions représentatives transnationales émergent - en Europe essentiellement ${ }^{30}$ , mais elles se heurtent à l'absence d'organisation patronale et aux difficultés pour les organisations professionnelles de dépasser le cadre national ${ }^{31}$. Par conséquent, les institutions «traditionnelles » cèdent la scène internationale à un ensemble d'acteurs dont la particularité est d'être souvent extérieurs aussi bien au cadre national qu'au secteur d'activité. Avec plus d'acuité que dans le contexte hexagonal $^{32}$, la perspective internationale des conflits du travail met en lumière la porosité entre mondes associatifs et professionnels, auxquels viennent s'ajouter très souvent des institutions internationales $^{33}$. L'échelle internationale invalide-t-elle pour autant l'approche par les relations professionnelles ? En tout cas, malgré ses difficultés, l'action syndicale perdure, et l'émergence progressive de nouvelles «méga » structures ${ }^{34}$, dont l'implication dans des négociations récentes ${ }^{35}$ ou des actions internationales ${ }^{36}$ a des répercussions nationales et mondiales, nous incite à redéfinir le champ de ces relations professionnelles - ses acteurs, leurs rôles et leurs interactions - plutôt qu'à l'abandonner.

Un enjeu des relations internationales

Enfin, l'approche internationale des conflits du travail nous amène à reconsidérer la dimension politique de cet objet. Depuis la chute du Mur et l'effondrement de l'Union Soviétique, la perception

décembre 2014) ; pour le Guatemala, Quentin Delpech, Mobilisations syndicales et violences au Sud, Paris, Karthala, 2014.

30. Élodie Béthoux, «Vers une représentation européenne des salariés. Les comités d'entreprise européens face aux restructurations », Sociologie du travail, 51, 2009, p. 478-498.

${ }^{31}$. François Aballéa, Arnaud Mias (dir.), Mondialisation et recomposition des relations professionnelles : un état des lieux, Toulouse, Octares, 2010. Le cas des cheminots européens constitue une bonne illustration de cette situation, malgré des tentatives de mobilisations transnationales. Hilal Nadia, «L'Europe, nouvelle figure de la crise syndicale : les syndicats face à la libéralisation du rail en Europe », Politique européenne, 27, 2009, p. 75-103.

32. J.-E. Rey, « Nouveaux conflits collectifs dans notre société de réputation : de la grève interne à l'action collective externalisée », cité ; I. Sommier, «Vers une approche unifiée de la conflictualité sociale », cité.

${ }^{33}$. Comme dans le cas de la catastrophe du Rana Plaza : un accord a été signé ensuite, le 15 mai 2003dans les locaux de l'OIT à Genève entre des syndicats mondiaux (IndustriALL) et des marques et entreprises donneuses d'ordre, avec pour signataire témoin un collectif d'ONG.

34. Anne Dufresne, «Naissance d'un euro-méga-syndicat. La fédération des industries européennes IndustriALL », Chroniques internationales de l'IRES, 141, juillet 2013, p. 21-34.

35. Depuis la signature de l'accord du Rana Plaza, IndustriALL a revendiqué en un an la syndicalisation de plus «de 40000 travailleuses et travailleurs dans 120 fabriques et ateliers » (http://www.industriallunion.org/fr/ premier-anniversaire-de-laccord-du-bangladesh).

${ }^{36}$. Au cours de l'été 2013, la Confédération syndicale internationale a mené une campagne de dénonciation des conditions de travail esclavagistes des ouvriers népalais sur les chantiers de la Coupe du monde prévue au Qatar en 2022. Après un premier reportage du Guardian faisant état de 44 morts en deux mois, la CSI a tenté de mobiliser en prédisant la mort de plus de 4000 personnes d'ici l'événement si rien n'était fait pour améliorer les conditions de travail. Bien qu'il n'ait fixé aucun calendrier, le Qatar a été contraint d'annoncer un assouplissement de ses conditions très restrictives d'emploi de la main-d'œuvre étrangère. 
profane et scientifique du travail comme espace de tensions sociales et arène de luttes démocratiques s'est considérablement étiolée. La place croissante des rhétoriques patriotiques ${ }^{37}$ et religieuses depuis le début des années 2000 ont en effet relégué la question sociale à l'arrière des scènes politiques nationales et internationales. À l'aube des années 2010, elle est réapparue dans les pays d'Afrique du Nord et du Moyen-Orient, régions pourtant «connues » aussi bien pour leurs nationalismes que pour leur ancrage religieux. Dans un contexte moins marqué par le ralentissement économique que par la «crise de l'État social autoritaire ${ }^{38}$, les révoltes qui ont agité ces régions ont été provoquées par les dérèglements des formes «traditionnelles » d'accès à l'embauche et par le tarissement des emplois disponibles ${ }^{39}$. Même si l'on ne peut réduire ces révoltes, ni dans leur genèse $^{40}$ ni dans les pays concernés ${ }^{41}$, à la seule problématique du travail, on peut considérer que celle-ci a été l'une des raisons majeures des transformations politiques survenues dans ces zones, ce qui fait de notre objet un véritable enjeu de relations internationales. Toutefois, les dimensions à la fois démocratiques et mondiales des conflits du travail ne se limitent pas aux cas emblématiques des soulèvements $\mathrm{du}$ «printemps arabe », elles se manifestent ailleurs, en particulier dans des pays pourvoyeurs de main-d'œuvre bon marché. En effet, la compétitivité de ces pays est très souvent rendue possible par une concentration des pouvoirs économiques et politiques qui se traduit, entre autres, par l'absence de protections et de droits aussi bien à l'intérieur qu'à l'extérieur de l'entreprise. Dès lors, il n'est pas rare que les grèves générales accompagnent les séquences électorales et que les revendications salariales se mêlent aux mots d'ordre politiques, comme au Cambodge ou au Bangladesh. Si l'on n'assiste pas ici, pour des raisons historiques et culturelles, à une contagion régionale, ces conflits n'en connaissent pas moins une internationalisation de leurs luttes qui, sous d'autres formes, ont des répercussions mondiales. En effet, à la suite de certaines catastrophes industrielles, les actions de responsabilisation des entreprises donneuses d'ordre menées par des entrepreneurs de mobilisations occidentaux quant aux conditions de travail des pays producteurs ont entraîné toute une série de conséquences, nationales et internationales. Bien qu'elles soient encore à l'état embryonnaire et donc perfectibles (Q. Delpech) ${ }^{42}$, ces campagnes produisent

\footnotetext{
${ }^{37}$. Françoise Daucé, Anne Le Huérou, Kathy Rousselet, «Les diversités du patriotisme contemporain », Critique internationale, 58, 2013, p. 9-17.

${ }^{38}$. Eberhard Kienle, Laurence Louër, "Comprendre les enjeux économiques et sociaux des soulèvements arabes », Critique internationale, 61, 2013, p. 11-17.

39. Allal Amin, «Trajectoires "révolutionnaires" en Tunisie », Revue française de science politique, 62 (5), 2012, p. 821-841. Parmi la mosaïque de profils que présente A. Allal, l'une des causes « ordinaires » de la révolution tunisienne a été le dysfonctionnement des pratiques clientélaires relatives aux questions de l'embauche. Compte tenu de son rôle, il serait bienvenu d'analyser finement le rôle de l'UGTT, principale centrale syndicale de Tunisie, dans l'élaboration de la nouvelle constitution du pays.

${ }^{40}$. E. Kienle, L. Louër, «Comprendre les enjeux économiques et sociaux des soulèvements arabes », art. cité. ${ }^{41}$. Myriam Catusse, «La question sociale aux marges des soulèvements arabes: leçons libanaises et marocaines », Critique internationale, 61, 2013, p. 19-34.

${ }^{42}$. Et P. Barraud de Lagerie, «L'affaire Spectrum », art. cité.
} 
des formes inédites, dans leurs formes ${ }^{43}$ comme dans leurs résultats, de régulation de la division internationale du travail qui restent à mesurer ainsi qu'à questionner. Et parce que ces conflits ne constituent, dans un marché mondial du travail qui demeure profondément anomique, que la partie «émergée » de ces mobilisations, il est plus que nécessaire que le travail redevienne, aux côtés de l'économie, du nationalisme et de la religion, un objet central dans l'intelligibilité des dynamiques actuelles des sociétés contemporaines ${ }^{44}$.

Maxime Quijoux est chargé de recherche au CNRS et membre du laboratoire Printemps (UMR 8085) de l'Université Versailles-Saint-Quentin-en-Yvelines. Ses travaux portent sur les mobilisations et les coopératives de travail en Argentine et en France. Il a publié Néolibéralisme et autogestion : l'expérience argentine (Paris, Éditions de l'IHEAL, 2011), et prépare actuellement un dossier intitulé «État, marché et travail en Amérique latine » pour la revue Problèmes d'Amérique Latine (septembre 2014). Il vient également d'achever un ouvrage intitulé Bourdieu et le travail (Rennes, Presses universitaires de Rennes, à paraître fin 2014).

mquijoux@gmail.com

\footnotetext{
${ }^{43}$. J.-E. Rey, « Nouveaux conflits collectifs dans notre société de réputation : de la grève interne à l'action collective externalisée », cité.

${ }^{44}$. Je remercie les auteurs de ces deux dossiers, les évaluateurs anonymes de Critique internationale et les membres de son comité de rédaction pour leur soutien et leur implication constante dans l'élaboration de ce projet. Je remercie en particulier Sophie Béroud et Annette Jobert pour leurs commentaires et leur lecture attentive de cette introduction, dont les propos n'engagent évidemment que leur auteur.
} 